\title{
Estratégia de ensino e avaliação do curso de extensão em Cultivo de Plantas Medicinais do Jardim Botânico do Rio de Janeiro
}

 \\ Fonseca $^{\text {a }}$; Jeferson A. Gonçalves ${ }^{a}$; Ulisses C. Souza ${ }^{a}$; Anna C. A. e Defaveri ${ }^{a}$; João \\ Carlos Silva ${ }^{\mathrm{a}, \mathrm{b}, \mathrm{c}}$; Mara Zélia de Almeida ${ }^{\mathrm{a}, \mathrm{b}}$; Sonia Cristina S. Pantoja ${ }^{\mathrm{a}}$ \\ ${ }^{\text {a }}$ Centro de Responsabilidade Socioambiental do Jardim Botânico do Rio de Janeiro, Rio de Janeiro, RJ - Brasil \\ ${ }^{\mathrm{b}}$ Farmácia da Terra, Universidade Federal da Bahia, Salvador, BA - Brasil \\ ${ }^{c}$ Programa de Pós-graduação em Ciências da Educação da Universidad Nacional de La Plata/Facultad de \\ Humanidades y Ciencias de la Educación - iCePeM, La Plata - Argentina
}

\section{Histórico do Artigo}

Recebido em:

$15 / 11 / 2017$

Aceito em:

$28 / 02 / 2018$

Palavras-chave:

Ensino-Aprendizagem;

Jardim Botânico;

etnobotânica.

Keywords:

Teaching and Learning; Botanical Garden; ethnobotany.

\begin{abstract}
RESUMO
Objetivou-se verificar o uso de espécies botânicas pelos estudantes do curso de extensão em "Técnicas de cultivo de plantas medicinas", do Centro de Responsabilidade Socioambiental do Jardim Botânico do Rio de Janeiro (CRS-JBRJ), como estratégia para melhoria do ensinoaprendizado e como base para a elaboração de um modelo de ensino sobre o tema. Para tanto, foi realizado um estudo exploratório descritivo com 35 alunos de ambos os sexos, das edições de 2016 e 2017, do curso através de um questionário estruturado, composto por dez questões. As plantas informadas pelos alunos foram utilizadas em aula, visando validar sua identificação. Foram citadas 48 espécies, com destaque para Matricaria chamomilla L. (n=13) e Cymbopogon citratus (D.C.) Stapf $(\mathrm{n}=11)$, e a infusão foi o uso relatado por todos. Em relação às plantas medicinais, os alunos informaram a maneira de obtenção, o motivo do uso, a frequência em que as utilizam em substituição a medicamentos alopáticos, a quantidade de espécies utilizadas simultaneamente e a credibilidade no tratamento e na possibilidade de toxidade decorrente da utilização. As relações expressas com os usos e elevado número de citações das etnoespécies levantadas demonstram uma alta difusão do conhecimento, importante para construção dinâmica estratégica em sala de aula e para potencializar a relação do ensino-aprendizado dos estudantes do curso. A elaboração curricular proposta neste estudo foi construída a partir do somatório da experiência estratégica de ensino e das experiências relatadas na literatura. Os dados fornecerão embasamento para a elaboração de oficinas, cursos de curta e longa-duração e especializações, bem como subsídio para um modelo de educação emancipatória, participativa e popular.
\end{abstract}

Teaching strategy and evaluation of the extension course in Medicinal Plants Cultivation of the Jardim Botânico do Rio de Janeiro

\section{ABSTRACT}

The use of botanical species by students of the extension course "Cultivation techniques of medicinal plants" of Centro de Responsabilidade Socioambiental do Jardim Botânico do Rio de Janeiro (CRSJBRJ) was assessed as a strategy for improving teaching-learning and as basis for elaborating a model on the subject. For that, a descriptive exploratory study was carried out with 35 students of both sexes from the 2016 and 2017 editions of the course. A ten questions structured questionnaire was used in the survey. The plants informed by the students were used in class to validate their identification. They cited 48 species, with emphasis on Matricaria chamomilla $\mathrm{L} .(\mathrm{n}=13)$ and Cymbopogon citratus (D.C.) Stapf $(\mathrm{n}=11)$. The use reported by all was the infusion. As regards medicinal plants, students reported on the method of collection, the reason for their use, the frequency with which they are used in place of allopathic medicines, the number of simultaneously used species, the credibility of the treatment and the possibility of toxicity arising from the use. The relationships between the uses and high number of ethnoespecies quotations demonstrate a high knowledge diffusion, important for strategic dynamic construction in the classroom and to enhance the teaching-learning relationship of students. The curricular elaboration proposed here was built from the sum of the strategic teaching experience and the literature. This data will provide support for workshops, short- and long-term courses and specializations, as well as for a model of emancipatory, participatory and popular education.

\footnotetext{
* Autor correspondente: ygorjesse@jbrj.gov.br (Y. J. Ramos)
} 


\section{Introdução}

No Brasil, as Práticas Integrativas e Complementares (PIC) foram incentivadas à incorporação no Sistema Único de Saúde (SUS), a partir da publicação da Política Nacional de Práticas Integrativas e Complementares (PNPIC), com as metas de reconhecer as práticas tradicionais populares e melhorar a adesão ao tratamento do paciente na atenção primária à saúde $(1,2)$.

As PICs, como formas de cuidados em saúde, concretizaram-se em um modelo que respeita questões sociais e estimula o autocuidado. No entanto, algumas experiências ainda encontram dificuldades nos "modos de fazer" de práticas como acolhimento, autonomia, integralidade e vínculo. Além disso, a falta de financiamento/contrapartidas e de interesse por parte de gestores, assim como o não interesse no aprendizado/falta de conhecimentos por parte dos profissionais, também são óbices que se apresentam na implantação desses serviços $(1,2,3,4)$.

Estudos apontam que os profissionais da saúde se sentem confiantes em indicarem os benefícios de PICs quando os utilizam ou conhecem veementemente, principalmente quando existem evidências científicas contundentes sobre essas práticas. A dificuldade em indicar o uso de PICs relaciona-se, dentre outras argumentações, com existência de sobreposição dos conhecimentos associados ao modelo de pensar saúde do sistema médico hegemônico. Como consequência, verificam-se dificuldades na visibilidade para o funcionamento/existência das práticas e no fortalecimento de modelos médicos holísticos, com mais conhecimento técnico e menos preconceito $(5,6)$.

Entre diversas técnicas em PICs que se fortaleceram no SUS nesses dez anos de incentivo, citamos a Fitoterapia, que, popularmente, no Brasil, fundamenta-se nos usos tradicionais de plantas medicinais, cuja origem está na diversificada herança étnicocultural, que, em muito, contribui para a promoção da saúde da população (7).

O atendimento seguro por parte dos profissionais da saúde está impreterivelmente vinculado aos seguintes fatores: o conhecimento prévio desse profissional sobre a identificação correta da espécie vegetal a ser indicada; a terapêutica seguida; a garantia da qualidade das plantas medicinais/drogas vegetais/preparações extemporâneas, verificando a não ocorrência da perda da efetividade e da segurança relacionadas aos seus princípios ativos; e a facilidade para identificações de possíveis riscos que possam estar associados a intoxicações causadas pelo uso inapropriado pelo paciente (8).

O reconhecimento por parte dos profissionais de saúde e a tentativa de aproximação do uso da fitoterapia no tratamento de seus pacientes/clientes pelo SUS configuram-se ainda como desafios, já que esses profissionais oferecem resistência à prática por serem formados para atuar no modelo médico hegemônico - que se sobrepõe nas ações e práticas de saúde de modelos holístico como o sobre citado (9).

Analisando que as diretrizes da PNPIC visam ao incentivo do tornar disponíveis plantas medicinais em suas diversas formas ao SUS, visualizamos o seu cultivo como ferramenta importante para aproximação desses pacientes/clientes pelo SUS, na construção dinâmica do conhecimento com os profissionais envolvidos. Esse modelo de interação para melhoria das relações (paciente/profissionais/plantas medicinais) já havia sido pensado na década de 80, com a criação das Farmácias Vivas pelo Professor José de Abreu Matos na Universidade Federal do Ceará, mas ainda existem algumas lacunas para avaliar essas interações $(1,10)$.

Pensando nesses gargalos, Silva et al. utilizaram uma metodologia da etnobotânica como estratégia de avaliação de ensino do curso de jardinagem para jovens do Programa Social, Educação e Trabalho do Jardim Botânico do Rio de Janeiro (JBRJ) (11). O objetivo dos autores foi entender o processo do conhecimento adquirido e a 
potencialização do modelo didático aplicado, demonstrando ser este um mecanismo interessante para responder à questão aberta.

Apesar da reconhecida importância da etnobotânica aplicada para a conservação e para o desenvolvimento sustentável, verificam-se, ainda, lacunas relacionadas ao uso de metodologias desta ciência para avaliar e atualizar os cursos ou programas de educação permanente em saúde, envolvendo plantas medicinais. Dentre os problemas associados, o principal é o desafio da inter e multidisciplinaridade, que requer a inserção de conceitos e métodos de várias disciplinas, algo ainda insuficientemente assimilado e pouco praticado pelos docentes brasileiros (11).

A relação ensino-aprendizagem traz a necessidade de avaliar o conhecimento preestabelecido pelo indivíduo no seu meio para incorporar metodologias que atendam a um modelo de construção dinâmica do conhecimento em sala de aula. Quando refletimos nos modelos de ensino de plantas medicinais, é imprescindível entender as relações dos seus usos para alívios de agravos e danos à saúde.

Visando essa integração de avaliar o curso e contribuir com a questão da ampliação do uso de PICs, objetivou-se verificar as relações de usos de espécies botânicas mais utilizadas pelos estudantes do curso de extensão em "Técnicas de Cultivo de Plantas Medicinais", do Centro de Responsabilidade Socioambiental (CRS) do JBRJ, Ademais, a partir desses dados, propor bases para a criação de modelos de ensino de cultivo das plantas medicinais para os diferentes níveis de atores sociais e setores interrelacionados, constituindo estratégia e ferramenta para avaliação desse modelo didático.

\section{Material e Método}

\subsection{Caracterização da área e da população em estudo}

A atividade foi realizada com 35 estudantes do curso de extensão intitulado "Técnicas de Cultivos de Plantas Medicinais", ministrado semanalmente em dez aulas no CRSJBRJ, situado no JBRJ, na zona sul da cidade do Rio de Janeiro, estado do Rio de Janeiro, Brasil, com as seguintes coordenadas geográficas: $22^{\circ} 57^{\prime} \mathrm{N}$ e $-43^{\circ} 13^{\prime} \mathrm{L}$. Todos os estudantes eram moradores da região urbana da cidade do Rio de Janeiro. No CRS, estão centralizadas todas as atividades ligadas à responsabilidade socioambiental do JBRJ e a zona didática de cultivo desde 1912 (12). O CRS atua na área de educação (12), desenvolvendo cursos de extensão com diversas vertentes, envolvendo a biodiversidade. Destaca-se o fato de que não foram encontrados na literatura relatos de cursos com tal temática.

\subsection{Delineamento do estudo}

O estudo baseou-se em duas etapas: a primeira delas consistiu em atividades desenvolvidas com os estudantes para avaliar o modelo de ensino-aprendizado do curso de extensão. Nessa etapa, os estudantes foram abordados, inicialmente, com a apresentação do projeto de ensino, seguida de uma breve apresentação acerca do seu objetivo. Os estudantes foram convidados a participar da aplicação de um questionário inicial, com finalidade de obter informações sobre os usos de plantas medicinais, a preencher formulários individuais a cada dia de aula concluída e a participar de uma autoavaliação coletiva, cuja finalidade dos formulários diários e da autoavaliação é obter as percepções sobre a metodologia e verificar as evoluções realizadas na aplicação do modelo. Parte dos resultados referentes às respostas obtidas junto aos estudantes foram transformados em porcentagem. 
A segunda etapa, que se baseou na experiência obtida, preceitos levantados previamente e revisão da literatura, consistiu na elaboração de uma proposta de base curricular para cursos de cultivo de plantas medicinais e no amparo estratégico das boas avaliações de funcionamento.

\subsection{Coleta dos dados}

Como instrumento de coleta de dados inicial, foi utilizado um questionário estruturado, composto por dez questões e aplicado a cada edição do curso (no segundo semestre de 2016 e no primeiro semestre de 2017), no primeiro dia de aula. Para construção do questionário, foram considerados: dados referentes à faixa etária e nível de escolaridade dos estudantes; questões relacionadas às suas práticas com plantas medicinais, tais como usos, obtenção e associações. Foram utilizadas duas listas livres, uma para citar as espécies medicinais, suas partes usadas e formas de preparos; e a outra para mencionar plantas medicinais que apresentavam registro de reações adversas $(13,14,15)$.

A partir do relato dos alunos, foram tabulados os nomes populares das etnoespécies. Como método de validação das etnoespécies, alguns exemplares dessas possíveis espécies foram apresentados aos estudantes em dois momentos, os quais ocorreram na terceira e na décima aula. A etnoespécie foi considerada validada quando houve o seu reconhecimento por parte dos alunos que a citaram. Para a identificação das espécies que não puderam ser apresentadas aos alunos e, por se tratar de alunos residentes em área urbana, foram consideradas referências comerciais como sachês, comprimidos, óleos, tinturas, cremes e pomadas. Com a finalidade de selecionar opções para a identificação das etnoespécies, foram consultados os seguintes documentos oficiais: farmacopeia Brasileira (16), Instrução Normativa $n^{\circ}$ 5, de 11 de dezembro de 2008 (17), Carvalho et al. (18) e a Resolução da Diretoria Colegiada (RDC) $\mathrm{n}^{\circ} 26$ de 13 de maio de 2014 (19) - os quais contêm relações de plantas interessantes/potenciais do ponto de vista comercial. Para o tratamento taxonômico, foi consultado o Banco de Dados da Flora Brasileira - JABOT, do JBRJ (20), e o sistema Angiosperm Phylogeny Group IV (21).

A lista de etnoespécies validadas foi então comparada às encontradas no Memento Terapêutico do Estado do Rio de Janeiro (MT-RJ) (22) e na Relação Nacional de Plantas de Interesses para o Sistema Único de Saúde (RENISUS) (23).

Os formulários diários eram compostos por dez perguntas, com finalidade de avaliar a qualidade do material didático, a didática do professor, o desenvolvimento da metodologia aplicada durante as aulas e as dificuldades observadas. A autoavaliação realizada no final do curso ocorreu na forma de diálogo em grupo.

\subsection{Elaborações das bases dos modelos curriculares de ensino de cultivo de plantas medicinais}

Para elaborar bases para a construção de modelos curriculares de ensino, no sentido de desenvolver/aperfeiçoar técnicas e cuidados para a produção e o cultivo de plantas medicinais, entendemos que esta prática deve estar lastreada nos preceitos teóricos da psicogenética de Piaget (24), na aprendizagem significativa de Ausubel (25), na teoria de Jung $(26 ; 27)$ e na pedagogia de Freire (28). As referidas teorias, bem como os teóricos, fundamentam que o desenvolvimento da matriz conceitual curricular deve ancorar-se no conhecimento pré-existente do participante. Trata-se de um esquema que deverá possuir um método participativo, dinâmico e multidisciplinar. Tal metodologia 
também se apoiou nos quatro pilares da educação, segundo a Organização das Nações Unidas para a Educação, a Ciência e a Cultura (UNESCO): Aprender a ser, aprender a conviver, aprender a fazer e aprender a aprender. Além disso, foram adotados os atores sociais definidos por Secchi (29), contemplando os níveis de atendimento da Atenção Primária em Saúde, Farmácias Vivas, setores produtivos de plantas medicinais e dados para elaboração de currículo preconizado pelo Ministério da Educação. Para subsidiar a proposta, também foram consideradas as pesquisas consultadas.

\section{Resultados}

O grupo entrevistado constituiu 35 alunos, dos quais $66 \%$ e $34 \%$ são representantes do sexo feminino e masculino, respectivamente. A faixa etária entre 18 e 27 anos foi a de maior representatividade, somando 52\%; seguida das faixas entre 28 e 37 anos, com 17\%; entre 58 e 47 anos, com 14\%; entre 48 e 57, com 11\%; e 6\% com mais de 58 anos. Em relação à escolaridade dos estudantes, 31\% apresentaram Ensino Superior incompleto, 29\% com Ensino Superior completo e os demais com menor escolaridade.

A partir do relato dos alunos e das consultas aos bancos de dados e referências, foram citadas 48 etnoespécies, as quais se encontram listadas na Tabela 1. Após classificação e determinação, seguindo a metodologia adotada no estudo, a família Lamiaceae obteve seis etnoespécies com 22 citações, seguida da família Asteraceae com cinco etnoespécies e 21 citações e Poaceae com duas etnoespécies e 12 citações.

Entre as etnoespécies, a mais citada foi Matricaria chamomilla L., mencionada 13 vezes com indicações de uso para o tratamento de cólicas e como calmante, seguida de Cymbopogon citratus (DC) Stapf., apontado 11 vezes para o tratamento de "dores de cabeça, digestão e como relaxante".

(Parei)Quando questionados sobre como obtêm as plantas medicinais, 25\% dos estudantes responderam que as compram em feiras livres ou erveiros, $17 \%$ alegaram comprar na forma industrializada, $10 \%$ declararam que extraem diretamente da natureza, $9 \%$ fazem uso de espécies extraídas dos quintais das suas casas e $7 \%$ as adquiriram através de vizinhos.

Com relação às situações em que fazem usos das plantas medicinais, 29\% afirmaram uso somente em casos de doença, seguidos de $26 \%$ que alegaram uso diário, mesmo não havendo problemas de saúde, e de $19 \%$ cujo uso é preventivo.

Quando interrogados sobre a frequência com que substituem medicamentos alopáticos por plantas medicinais, $26 \%$ afirmaram fazê-lo frequentemente, seguidos de $20 \%$ que alegaram praticar essa substituição às vezes e de $3 \%$ que alegaram uso concomitante com os medicamentos.

Foram questionados também sobre a quantidade de espécies de plantas medicinais costumam utilizar juntas: $27 \%$ confirmaram o uso de duas plantas concomitantemente, $11 \%$ alegaram uso de até três ervas medicinais, $11 \%$ alegaram nunca misturar e $3 \%$ afirmaram uso de "garrafadas".

Quando questionados se acreditam no poder terapêutico das plantas medicinais, 63\% afirmaram positivamente e $37 \%$ não souberam informar. Dentre os motivos para acreditarem ou não na eficácia do uso de plantas medicinais foram citados: "Comprovação científica e tradicional; Por ser natural; Compostos bioativos; São econômicos; Depende da fonte de informação; Uso pelos povos tradicionais; Conhecimento prático pessoal e; Devido à obtenção dos resultados esperados durante o uso".

Quando questionados se determinada espécie botânica, mesmo sendo medicinal, também poderia apresentar toxidade, 19\% afirmaram acreditar nessa possibilidade, $65 \%$ 
afirmaram não acreditar e $16 \%$ não souberam informar.

Ainda sobre a questão de riscos no uso de plantas medicinais, quando interrogados sobre o conhecimento de casos por intoxicação por uso, $86 \%$ dos estudantes afirmaram desconhecer qualquer ocorrência sobre o assunto e 14\% afirmaram ciência de casos. Foram citados os seguintes casos, efeito adverso e motivo: "sene - diarreia (tomou em excesso); citronela - ingestão (confundiu com capim-limão); copaíba e melaleuca (vermelhidão na pele); chá verde - queda de pressão arterial; casca do abacaxi diarreia; excesso de chás - alergias e taquicardia; chá de hibisco - hipotensão (tomou em excesso); boldo - problemas hepáticos (tomou em excesso) e; canela - abortiva.

Como pergunta final, o questionário inquiriu sobre a indicação do uso de plantas medicinais na cura de doenças para outras pessoas. Como resposta, 54\% afirmaram que indicam, 6\% alegaram não indicar e 40\% não souberam informar.

Foi percebido nas avaliações dos formulários sobre a dinâmica da aula que houve envolvimento expressivo da turma com a introdução das espécies citadas, já que estão inseridas em suas práticas de saúde cotidianas.

Tendo em vista essa necessidade de incorporar à prática dos outros e um modelo de ensino voltado para pedagogia emancipadora, seguindo metodologia foi efetuada a elaboração dos modelos curriculares de ensino nos cursos baseados em relatos de experiências, atividades extensionistas e demais relatos da literatura. Esses resultados estão sumarizados na Figura 1.

Tabela 1: Relação de espécies botânicas citadas como plantas medicinais utilizadas pelos estudantes, parte utilizada, forma de preparo e a finalidade do seu uso. Legenda: *=identificação baseada em consulta às legislações: Farmacopeia Brasileira (16), Instrução Normativa $n^{\circ} 5$ De 11 de dezembro de 2008 (17), Carvalho et al. (18) e RDC nº 26 de 13 de maio de 2014 (19); NI=não informado; \#=não identificada; E1 e E2=citações na edição 1 e na edição 2 do curso de extensão, respectivamente; MT-RJ=Memento Terapêutico do Rio de Janeiro (27); e R=Relação de Plantas Medicinais de Interesse para o Sistema Único de Saúde, RENISUS (28); x=Registro encontrado.

\begin{tabular}{|c|c|c|c|c|c|c|c|c|c|}
\hline \multirow{2}{*}{ FAMÍLIA } & \multirow{2}{*}{ NOME CIENTÍFICO } & \multirow{2}{*}{$\begin{array}{c}\text { NOME } \\
\text { VERNACULAR }\end{array}$} & \multirow{2}{*}{$\begin{array}{c}\text { PARTE } \\
\text { UTILIZADA }\end{array}$} & \multirow{2}{*}{$\begin{array}{l}\text { FORMA DE } \\
\text { PREPARO }\end{array}$} & \multirow{2}{*}{$\begin{array}{l}\text { INDICAÇÕES } \\
\text { TERAPÊUTICAS }\end{array}$} & \multicolumn{2}{|c|}{ CITAÇÃO } & \multirow{2}{*}{ MT - RJ } & \multirow{2}{*}{$\mathbf{R}$} \\
\hline & & & & & & E1 & $\mathbf{E 2}$ & & \\
\hline ANACADIACEAE & Schinus terebinthifolius Raddi. & aroeira & folha & Banho & anti-inflamatório & 1 & & & \\
\hline \multirow{2}{*}{ APIACEAE } & Coriandrum sativum $\mathrm{L}$. & coentro & folha / semente & infusão / fervura & anti-inflamatório & & 2 & & \\
\hline & Pimpinella anisum $\mathrm{L} . *$ & erva-doce & semente & fervura & relaxante & 1 & 2 & $\mathrm{x}$ & \\
\hline AQUIFOLIACEAE & Ilex paraguariensis A.St.-Hil.* & mate & folha & infusão & NI & & 1 & & \\
\hline ASPHODELACEAE & Aloe vera (L.) Burm. $\mathrm{f}$. & babosa & folha / seiva & in natura / gel & $\begin{array}{c}\text { queimaduras / } \\
\text { cicatrizante / hidratante }\end{array}$ & & 4 & & $\mathrm{x}$ \\
\hline \multirow{5}{*}{ ASTERACEAE } & Matricaria chamomilla $\mathrm{L} . *$ & camomila & flor / folha & infusão & calmante / cólica & 4 & 9 & & \\
\hline & Arnica montana $\mathrm{L} . *$ & arnica & extrato vegetal & infusão / pomadas & $\begin{array}{l}\text { anti-inflamatório / } \\
\text { dores / contusões }\end{array}$ & 1 & 2 & $\mathrm{x}$ & \\
\hline & Mikania glomerata Spreng. & guaco & folha & xarope & $\begin{array}{l}\text { contra tosse / alergia } \\
\text { respiratória / } \\
\text { expectorante }\end{array}$ & 1 & 2 & $\mathrm{x}$ & \\
\hline & Calendula officinalis L.* & calêndula & NI & pomada & feridas & & 1 & $\mathrm{x}$ & $\mathrm{x}$ \\
\hline & Baccharis trimera (Less.) DC. & carqueja & NI & NI & digestivo & & 1 & $\mathrm{x}$ & $\mathrm{x}$ \\
\hline BORAGINACEAE & Varronia curassavica Jacq.* & erva-baleeira & folha & tintura / infusão & $\begin{array}{l}\text { anti-inflamatório / } \\
\text { analgésico / } \\
\text { reumatismo }\end{array}$ & & 2 & $\mathrm{x}$ & $\mathrm{x}$ \\
\hline BROMELIACEAE & Ananas comosus (L.) Merril* & abacaxi & casca & fervura & diurético & & 1 & & $\mathrm{x}$ \\
\hline CARYOPHYLLACEAE & Dianthus caryophyllus $\mathrm{L}$. & cravo & NI & NI & anti-inflamatório & 1 & & & \\
\hline CELASTRACEAE & $\begin{array}{l}\text { Maytenus ilicifolia Mart. ex } \\
\text { Reissek* }\end{array}$ & espinheira-santa & folha & fervura & dores no estômago & & 1 & $\mathrm{x}$ & $\mathrm{x}$ \\
\hline CRASSULACEAE & $\begin{array}{c}\text { Kalanchoe brasiliensis } \\
\text { Cambess. }\end{array}$ & saião & folha & maceração & resfriado / dor muscular & & 2 & & \\
\hline EQUISETACEAE & Equisetum arvense $\mathrm{L}$. & cavalinha & folha & infusão / tintura & reumatismo & & 1 & & $\mathrm{x}$ \\
\hline
\end{tabular}


Vittalle - Revista de Ciências da Saúde v. 30, n. 1 (2018) 168-181

\begin{tabular}{|c|c|c|c|c|c|c|c|c|c|}
\hline & & & & & & & \multicolumn{3}{|c|}{ Continuação } \\
\hline \multirow{2}{*}{ FAMÍLIA } & \multirow{2}{*}{ NOME CIENTÍFICO } & \multirow{2}{*}{$\begin{array}{c}\text { NOME } \\
\text { VERNACULAR }\end{array}$} & \multirow{2}{*}{$\begin{array}{c}\text { PARTE } \\
\text { UTILIZADA }\end{array}$} & \multirow{2}{*}{$\begin{array}{l}\text { FORMA DE } \\
\text { PREPARO }\end{array}$} & \multirow{2}{*}{$\begin{array}{l}\text { INDICAÇÕES } \\
\text { TERAPÊUTICAS }\end{array}$} & \multicolumn{2}{|c|}{ CITAÇÃO } & \multirow{2}{*}{ MT - RJ } & \multirow{2}{*}{$\mathbf{R}$} \\
\hline & & & & & & $\mathbf{E 1}$ & $\mathbf{E 2}$ & & \\
\hline \multirow{2}{*}{ FABACEAE } & $\begin{array}{l}\text { Stryphnodendron adstringens } \\
\text { (Mart.) Coville* }\end{array}$ & barbatimão & casca / folha & infusão & infecção vaginal & & 1 & & $\mathrm{x}$ \\
\hline & Copaifera langsdorffii Desf. & copaíba & óleo & in natura & \begin{tabular}{|c|} 
machucados / infecções \\
internas / cicatrizante
\end{tabular} & & 2 & & $x$ \\
\hline GINKGOACEAE & Ginkgo biloba $\mathrm{L} . *$ & ginko-biloba & folha & infusão & memória & & 1 & & \\
\hline IRIDACEAE & Crocus sativus $\mathrm{L} . *$ & açafrão & raiz & fervura / in natura & $\begin{array}{c}\text { antioxidante / anti- } \\
\text { inflamatório }\end{array}$ & & 1 & & \\
\hline \multirow{7}{*}{ LAMIACEAE } & Plectranthus barbatus Andr. & boldo & folha & chá / infusão & $\begin{array}{c}\text { gastrite / dor estômago / } \\
\text { digestivo }\end{array}$ & 5 & 2 & $\mathrm{x}$ & $\mathrm{x}$ \\
\hline & Melissa officinalis L. & $\begin{array}{l}\text { erva-cidreira / } \\
\text { melissa }\end{array}$ & folha / galho & infusão & $\begin{array}{c}\text { cólica/relaxante/dor de } \\
\text { cabeça }\end{array}$ & 3 & 2 & $\mathrm{x}$ & \\
\hline & Mentha $\mathrm{x}$ piperita $\mathrm{L}$. & hortelã & $\begin{array}{l}\text { folha / óleo } \\
\text { essencial }\end{array}$ & $\begin{array}{c}\begin{array}{c}\text { diluição em óleo / } \\
\text { fervura / infusão / } \\
\text { chá }\end{array} \\
\end{array}$ & $\begin{array}{l}\text { resfriado / dor de } \\
\text { cabeça }\end{array}$ & 2 & 3 & & $\mathrm{x}$ \\
\hline & Rosmarinus officinalis $\mathrm{L}$. & alecrim & $\begin{array}{c}\text { óleo essencial / } \\
\text { folha / caule }\end{array}$ & $\begin{array}{c}\text { chá / inalação / } \\
\text { diluiçãao em óleo }\end{array}$ & $\begin{array}{c}\text { estimulante / } \\
\text { expectorante / queda de } \\
\text { cabelo }\end{array}$ & & 2 & & $x$ \\
\hline & Ocimum basilicum $\mathrm{L}$. & manjericão & folha & in natura & $\begin{array}{c}\text { problemas digestivos / } \\
\text { tempero }\end{array}$ & 1 & 1 & & \\
\hline & Lavandula angustifolia Mill. & lavanda & $\begin{array}{c}\text { óleo essencial / } \\
\text { flor / tudo }\end{array}$ & \begin{tabular}{|c|} 
inalação / diluição \\
em óleo
\end{tabular} & calmante & & 2 & & \\
\hline & Salvia officinalis $\mathrm{L}$. & sálvia & folha & infusão / chá & cabelo & & 1 & & \\
\hline \multirow[b]{2}{*}{ LAURACEAE } & Cinnamomum verum J.Presl* & canela & casca & fervura & cólica & & 2 & & \\
\hline & $\begin{array}{c}\text { Cryptocaria moschata Nees \& } \\
\text { Mart. ex Nees.* }\end{array}$ & noz-moscada & pó & NI & digestão & & 1 & & \\
\hline LILIACEAE & Allium sativum $\mathrm{L}$. & alho & raiz & in natura / fervura & antibiótico & & 1 & & $\bar{x}$ \\
\hline LYTHRACEAE & Punica granatum $\mathrm{L}$. & romã & casca & NI & $\begin{array}{l}\text { anti-inflamatório / } \\
\text { adstringente }\end{array}$ & 1 & & & $\mathrm{x}$ \\
\hline MONIMIACEAE & Peumus boldus Molina. & boldo do chile & folha & infusão & digestivo & & 1 & & \\
\hline MYRTACEAE & Eugenia uniflora $\mathrm{L}$. & pitanga & folha & infusão & febre & & 1 & & $\bar{x}$ \\
\hline OLEACEAE & Jasminum officinale $\mathrm{L} . *$ & jasmin & flor & infusão & NI & & 1 & & \\
\hline PASSIFLORACEAE & Passiflora edulis Sims & maracujá & folha / galho & fervura / infusão & relaxante & & 2 & $\mathrm{x}$ & $\bar{x}$ \\
\hline \multirow{2}{*}{ POACEAE } & $\begin{array}{l}\text { Cymbopogon citratus (DC) } \\
\text { Stapf. }\end{array}$ & capim-limão & folha & fervura / infusão & $\begin{array}{l}\text { relaxante / dor de } \\
\text { cabeça / digestão }\end{array}$ & 4 & 7 & & \\
\hline & $\begin{array}{l}\text { Cymbopogon winterianus } \\
\text { Jowitt. }\end{array}$ & citronela & óleo essencial & NI & repelente & & 1 & & \\
\hline \multirow{2}{*}{ RUTACEAE } & Citrus aurantium $\mathrm{L} . *$ & laranja & casca seca & infusão & digestivo & & 1 & & \\
\hline & Citrus x limon (L.) Osbeck* & limão & fruto & infusão & gripe & & 1 & & \\
\hline TURNERACEAE & Turnera cearensis Urb. & malva & folha & infusão & digestão & & 1 & & \\
\hline VERBENACEAE & $\begin{array}{l}\text { Lippia alba (Mill.) N.E.Br. ex P. } \\
\text { Wilson. }\end{array}$ & erva-cidreira & folha & infusão & dor de cabeça & & 1 & $\mathrm{x}$ & \\
\hline \multirow{2}{*}{ ZINGIBERACEAE } & Zingiber officinale Roscoe & gengibre & rizoma & $\begin{array}{c}\text { infusão / decocção } \\
\text { / in natura }\end{array}$ & $\begin{array}{l}\text { digestivo / dor de } \\
\text { garganta }\end{array}$ & 1 & 4 & & $x$ \\
\hline & Curcuma longa $\mathrm{L}$. & cúrcuma & rizoma & em pó & $\begin{array}{c}\text { anti-inflamatório / pasta } \\
\text { de dente }\end{array}$ & & 2 & $\mathrm{x}$ & $\mathrm{x}$ \\
\hline$\#$ & $\#$ & pimenta & NI & NI & resfriado & 1 & & & \\
\hline ALISMATACEAE & $\begin{array}{l}\text { Echinodorus macrophyllus } \\
\text { (Kunth) Micheli }\end{array}$ & chapéu-de-couro & folha & infusão & diurético & & 1 & $\mathrm{x}$ & \\
\hline \multirow{3}{*}{ THEACEAE } & Camellia sinensis (L.) Kuntze* & $\mathrm{NI}$ & folha & infusão & estimulante & & 1 & & \\
\hline & Camellia sinensis (L.) Kuntze* & chá-preto & (sachê) & infusão & digestivo & & 1 & & \\
\hline & Camellia sinensis (L.) Kuntze* & chá-verde & (sachê) & infusão & emagrecimento & & 1 & & \\
\hline
\end{tabular}



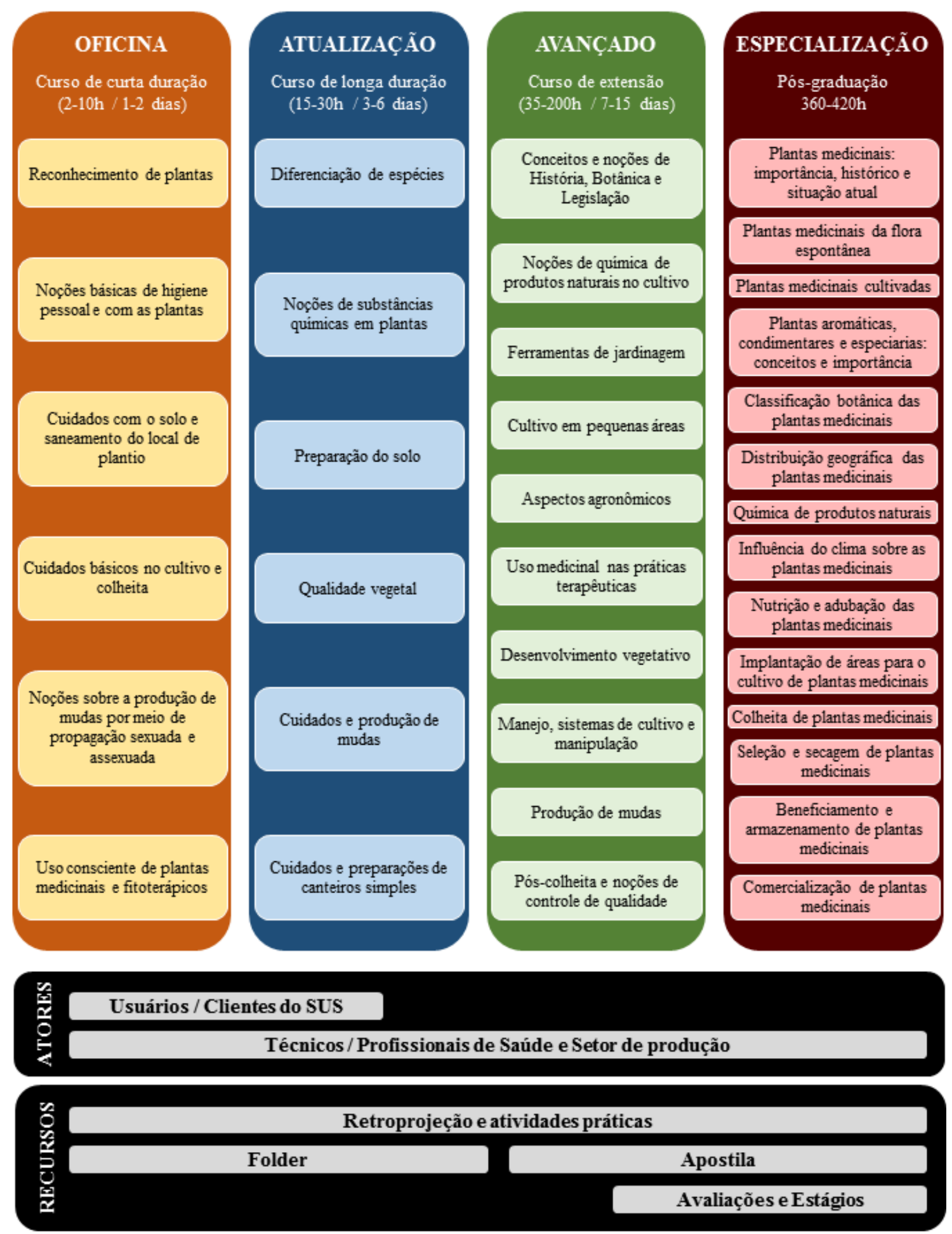

Figura 1: Grade curricular experimental proposta para o ensino sobre o cultivo de plantas medicinais para diferentes níveis de escolaridade, com seus respectivos atores e recursos.

\section{Discussão}

As espécies mais citadas foram a Matricaria chamomilla L. $(\mathrm{n}=13)$ e o Cymbopogon citratus (D.C.) Stapf $(\mathrm{n}=11)$, que também ganharam destaque nos estudos de etnobotânica urbana de Queiroz (30) e Viganó et al. (31), nos quais as famílias Asteraceae, Lamiaceae e Poaceae foram as mais representativas. Segundo Bennett; Prance (32), todas as famílias mencionadas são constituídas de plantas (muitas delas 
medicinais) que predominam em regiões tropicais, sendo que a maioria é de fácil propagação e porte relativamente pequeno, facilitando o cultivo em pequenas áreas e ambientes de condições nutricionais e ecológicas restritas.

A partir do relato de alunos de ambas as turmas foram detectadas coincidências de citação para 10 etnoespécies. Após essa percepção, foi adotada uma estratégia importante para a diminuição dos casos de intoxicação, no sentido de trabalhar esses indivíduos no processo de identificação correta das espécies botânicas medicinais que utilizam. Para espécies adquiridas no comércio não foram adotadas abordagens para além do uso consciente de plantas medicinais e fitoterápicos. Outra argumentação significativa é que a repetição de citação da espécie associada ao mesmo uso específico ou padrão de uso pode ser um mecanismo interessante para validação da aplicabilidade no tratamento $(7,9,33,34,35)$.

As análises em etnobotânica urbana podem aduzir parâmetros vultosos para estimar o potencial de uso de plantas medicinais, auxiliar a identificar possível toxicidade e averiguar sua relevância como ornamentais. A prática de ensino visando à diminuição de agravo demonstrou-se interessante, principalmente para faixa etária 18 a 27 anos, com maior representatividade no estudo, cujos integrantes demonstraram-se seguros no uso desses recursos terapêuticos (11).

Do ponto de vista da formação, estes são parâmetros interessantes a serem trabalhados em todos os níveis de conhecimento apresentados na Figura 1, pois um dos principais problemas relacionados ao uso errôneo/não consciente de plantas medicinais e fitoterápicos está relacionado à falta de compreensão das sintomatologias das doenças em questão e a não identificação adequada das espécies e de usos (33). Dessa forma, para atender a essa demanda pode-se sugerir a oferta de disciplinas com o tema reconhecimento de espécies vegetais e diferenciação de espécies vegetais, tais como, por exemplo, capim-limão e citronela.

Observando a Tabela 1 na comparação dos documentos oficiais, foram encontradas 13 espécies citadas no Memento Terapêutico do Rio de Janeiro e 18 na RENISUS. Somando a ocorrência das espécies em uma ou outra lista, foram encontradas 24 plantas, representando $50 \%$ do total listada. Os resultados sugerem a possibilidade de as espécies serem frutos do processo de disseminação difusa dos conhecimentos, fenômeno relacionado ao alto índice de comercialização, às relações ambiente-homemsaúde e à popularização de uso em áreas urbanas, derivado dos meios de comunicação e globalização $(11,34,36)$.

Em sistemas urbanos, os seres humanos estão habituados a ouvir sobre "progresso do conhecimento", porém, essa expressão não deve ser usada sem ser uma metonímia, principalmente no caso do que chamamos de conhecimento difuso. Este último ocorre quando o indivíduo se utiliza de sua tradição, de sua cultura, para difundir uma ideia, um saber, isto é, quem utiliza esta estratégia não "cria" (a não ser uma cultura em outros indivíduos), mas sim resgata o que já foi criado para progredir, intelectualmente falando ou não. Normalmente acontece em comunidades pequenas, entre familiares e entre grupos religiosos, nos quais não é necessário ter um nível de escolaridade mais elevado que os demais $(34,37)$.

Em ambas as edições dos cursos de extensão, ao trabalhar o levantamento de espécies com os estudantes, foi percebido que os indivíduos trouxeram sua cultura, seu conhecimento, sua tradição, o que na maioria das vezes não é uma prática comum nos sistemas urbanos. Para alguns autores esse processo de interseção e transação dos conhecimentos, conhecido como hibridização, é o principal responsável pela multiculturalidade presente nas cidades, que são sobreposições de fatores econômicos e culturais $(11,34,36)$. 
Os resultados obtidos na Tabela 1 foram de suma importância para a construção da metodologia de trabalho e das aulas, pois, como optamos por um processo de difusão do conhecimento e aplicação de um ambiente interativo, possibilitamos a aproximação entres os estudantes e a oportunidade dos professores vivenciarem essa troca de saberes, e de aproximarem-se de sua própria prática.

A difusão do conhecimento da utilização de plantas medicinais acaba tornando-se comum, mas o cultivo ainda constitui um gargalo como modelo intermediário nas PICs. Essa atividade proporciona aos atores ter contato com as espécies que fazem uso antes e/ou depois da prescrição pelos profissionais habilitados. A Figura 1 representa e exemplifica esse modelo em vários níveis.

A literatura possui diversos relatos de experiências e artigos relacionados a cada nível demonstrado. Ceolin et al. (38), por exemplo, realizaram um trabalho de atualização para 41 profissionais da área de saúde e foram desenvolvidas atividades práticas e teóricas que abordavam temas como: políticas públicas em relação às plantas medicinais e aos fitoterápicos; terapias complementares; informantes folk em plantas medicinais no Sul do Brasil; cuidados para o bom uso de plantas medicinais (coleta, preparação, conservação e uso); controle de qualidade; identificação taxonômica das plantas; cuidados na coleta e acondicionamento para montagem de um herbário; montagem das exsicatas; identificação das propriedades terapêuticas - princípios ativos e plantas tóxicas; relato de experiência dos municípios de São Lourenço do Sul, Rio Grande do Sul e Nova Petrópolis, Rio Grande do Sul; diferentes fitoterápicos para tratamento de feridas; e estudos etnobotânicos e clínicos sobre plantas medicinais, promovendo o segundo contato dos profissionais da atenção primária dos municípios da $3^{\mathrm{a}}$ Coordenadoria Regional de Saúde do Rio Grande do Sul com a prática e/ou educação permanente em saúde/curso para prescritos e jardineiros.

No trabalho de Damian et al. (39), os autores apontaram que a grade curricular do ensino superior é deficiente de uma formação técnico-prática em relação à fitoterapia durante todo o ramo acadêmico/profissional, o que acarreta em uma hesitação para a récipe da fitoterapia nos serviços de atenção primária e para orientação aos usuários sobre a sua utilização. Sendo assim, a prescrição de fitoterápicos e plantas medicinais, por parte dos profissionais, poderia ser estimulada a partir do processo de educação continuada e permanente dos profissionais no cotidiano de trabalho das equipes de saúde através dos cursos de especialização e capacitação. Logo, os profissionais capacitados poderiam diferenciar as plantas medicinais/fitoterápicos mais utilizados entre os pacientes que atendem e orientá-los, evitando práticas inadequadas que conduzam ao uso irracional, à crença nas propagandas midiáticas, às iatrogenias clínicas e à falta de adesão ao tratamento. No Brasil a Associação Brasileira de Fitoterápicos (Abfit) e a Universidade Aberta do SUS (UNA-SUS) são exemplos de instituições de ensino que oferecem cursos para capacitação e especialização na área de fitoterápicos.

As oficinas sobre plantas medicinais têm sido apontadas como grande estratégia didática principalmente na área da biologia por transitarem entre os saberes autorizados e não autorizados, tais como ciência com etnoconhecimentos, artes e práticas diversas, ampliando a perspectiva de continuidade/descontinuidade entre os chamados "saberes tácitos/saberes explícitos" para a análise das estratégias de poder que calam certos discursos e jogam no esquecimento certas práticas, assimilam, modificando algumas, e opacificam outras tantas. A perspectiva não disciplinar, iluminando a prática das oficinas, rompe com o funcionamento escolar disciplinar do tempo porque a oficina não cabe nos ritmos e rotinas temporais da educação formal. Uma oficina pode durar mais ou menos tempo em função dos desdobramentos que podem ocorrer, ao mesmo tempo em que há a possibilidade de intervalo, este é ditado pela ocasião. Rompe também com 
a separação disciplinar entre trabalho e lazer, porque oficina é essencialmente trabalho diversificado com prazer, e não pode ser reduzida à sucessão de tarefas rotineiras que caracteriza o chamado trabalho escolar. Finalmente, e mais importante de tudo que já foi exposto, rompe com a organização curricular disciplinar dos conteúdos. Dessa forma a oficina não exige idades determinadas, sexos determinados, escolaridade determinada ou pré-requisito de conhecimento para os seus participantes. É um processo educativo que ignora a avaliação enquanto julgamento, e a política de verdade dos conteúdos escolares, segmentação do saber em conteúdo escolar, cientificidade como validação de tudo isso (40).

As atividades clássicas para serem pensadas em modelos de oficinas são ações pontuais em saúde como: campanha do uso consciente/racional de plantas medicinais e fitoterápicos, preparações extemporâneas e cultivo. Um dos exemplos é o trabalho de Pereira e Albiero (41), em que a realização de oficinas de aprendizagem com os Agentes Comunitários em Saúde das unidades básicas de saúde selecionadas, a fim de desenvolver habilidades quanto ao plantio; coleta; secagem; armazenamento e preparo correto de chás (infuso e decocto); tinturas; alcoolaturas e xaropes.

As possibilidades e dificuldades do ensino para todos os níveis de escolaridade apresentados, nos fazem refletir que pensar no outro como fonte do saber é um ato revolucionário, e a educação segundo Paulo Freire torna-se uma forma de ação que une as linguagens da crítica e da possibilidade, além de representar a necessidade de um comprometimento por parte dos educadores em tornar a reflexão e ação crítica parte de um projeto que não inclua apenas as formas de opressão, mas também desenvolva uma fé profunda permanente na luta para a superação das injustiças sociais e na busca da humanização da própria vida e não marginalização das práticas não hegemônicas, como as PIC $(11,28)$.

A educação sozinha não tem condições de construir uma sociedade emancipada. A exclusão social, a globalização econômica e as políticas neoliberais excludentes consolidam um capitalismo que amplia a capacidade de produção de mercadorias, acúmulo de capital e geração de riquezas nas mãos de um grupo minoritário - os opressores/dominadores. Nesse sentido, a educação "deve exercitar processos de emancipação individual e coletiva, estimulando e possibilitando a intervenção no mundo a partir de um sonho ético-político da superação da realidade injusta". As oficinais/cursos são pensadas como modelos de retorno/devoluções e baseadas nos conhecimentos da comunidade, e por entender que essa prática deve refletir diretamente nas necessidades e vivências dos atores envolvidos, tornando-os emancipados e não marginalizados na condição social de ser um outro (42).

A emancipação defendida por Freire contempla também o chamado multiculturalismo, onde o direito de ser diferente numa sociedade dita democrática, também deve propiciar o diálogo crítico entre as inúmeras culturas, com vistas à ampliação e a consolidação dos processos emancipatórios (28).

\section{Considerações finais}

Diante dos resultados obtidos, existe uma relação íntima entre a utilização, as citações de espécies botânica levantada e a alta difusão do conhecimento, principalmente se levarmos em consideração que em sua maioria as espécies botânicas estão presentes no memento terapêutico do Rio de Janeiro e no RENISUS, além de tais dados terem sido levantados através de um grupo de diversificada faixa etária. Tal fato é de suma importância para a construção da dinâmica em sala de aula e para potencializar a relação do ensino-aprendizado dos estudantes do curso sobre plantas medicinais. 
Através da prática pedagógica adotada pelo CRS durante o curso, acredita-se que todos os estudantes, mesmo que com diferentes níveis de escolaridade, tendem a possuir o mesmo nível de aprendizado (existe uma relação simbólica com o aprendizado, o aluno aprende ao ver sua planta na aula), pois são acolhidos pelo curso. Acredita-se também que tais iniciativas de capacitação de profissionais de saúde atuantes na atenção primária da saúde, sobre plantas medicinais, são obrigatórias para alicerçar os princípios e diretrizes do SUS porque propiciam a ampliação das práticas terapêuticas em busca da integralidade da assistência, além de proporcionar aos profissionais conhecimento sobre as plantas medicinais e as políticas governamentais em relação ao tema e troca de experiências entre os participantes. Nesse sentido, iniciativas como essa devem ser difundidas em outros municípios com o objetivo de capacitar profissionais, para que se aproximem da realidade cultural da população assistida e integrem o saber popular ao conhecimento científico, permitindo a aliança de saberes.

\section{Agradecimentos}

Os autores agradecem aos estudantes a disponibilidade em participar da construção desta metodologia.

Conflito de interesses: Os autores declaram não haver conflito de interesses.

\section{Referências}

1. Brasil. Ministério da Saúde. Portaria n. 971, de 3 de maio de 2006. Aprova a Política Nacional de Práticas Integrativas e Complementares (PNPIC) no Sistema Único de Saúde. Diário oficial da União, Brasília, n. 84, seção I p. 19, 04 maio 2006.

2. Tesser CD. Práticas complementares, racionalidades médicas e promoção da saúde: contribuições poucos exploradas Complementary practices, medical rationalities, and health promotion: some overlooked. Cad. saúde pública 2009; 25(8), 1732-1742.

3. Barros NFD. Política Nacional de Práticas Integrativas e Complementares no SUS: uma ação de inclusão. Ciênc. saúde coletiva 2006; 11(3), 850-850.

4. Azevedo ED, Pelicioni MCF. Práticas integrativas e complementares de desafios para a educação. Trab. Educ. Saúde 2011; 361-378.

5. Santos CM, Tesser DC. Um método para a implantação e promoção de acesso às Práticas Integrativas e Complementares na Atenção Primária à Saúde. Ciênc. saúde coletiva 2012; 17(11): 3011-3024

6. Schveitzer MC, Esper MV, Silva MJPD. Práticas Integrativas e Complementares na Atenção Primária em Saúde: em busca da humanização do cuidado. Mundo saúde 2012; 442-451.

7. Almeida MZ, Lessa GM, Queiroz MOR, Cardoso DN, Santos FA. Fitoterapia no SUS no estado da Bahia: contribuição para valorização do conhecimento e das práticas tradicionais na rede básica de saúde. Rev. Fitos 2013; 6 (01): 29-34.

8. Bruning RMC, Mosegui BGG, Vianna MMC. A utilização da fitoterapia e de plantas medicinais em unidades básicas de saúde nos municípios de Cascavel e Foz do Iguaçu-Paraná: a visão dos profissionais de saúde. Ciênc. saúde coletiva 2012; 17(10): 2675-2685.

9. Almeida MZ, Léda PH, Silva MQ, Pinto A, Lisboa M, Guedes MLM, Peixoto AL. Species with medicinal and mystical-religious uses in São Francisco do Conde, Bahia, Brazil: a contribution to the selection of species for introduction into the local Unified Health System. Rev. Bras. Farmacogn. 2014; 24(2): 171-184.

10. Matos FJA. Farmácias vivas: sistema de utilização de plantas medicinais projetado para pequenas comunidades. Fortaleza: Editora UFC, 2002.

11. Silva JC, Firme TP, Almeida MZ, Ramos YJ. Etnobotânica como ferramenta de avaliação na difusão dos conhecimentos: relação dos jovens e o programa socioambiental do JBRJ. Rev. Fitos 2015; 9(2): 113-128.

12. Ramos DRM, Peixoto AL. Os caminhos do ensino e extensão no Jardim Botânico do Rio de Janeiro. Rev. Ciênc. Ext. 2017; 13(2): 141-153. 


\section{Vittalle - Revista de Ciências da Saúde v. 30, n. 1 (2018) 168-181}

13. Albuquerque UP, Hanazaki N. As pesquisas etnodirigidas na descoberta de novos fármacos de interesse médico e farmacêutico: fragilidades e pespectivas. Rev. Bras. Farmacogn. 2006; 16(0), 678689.

14. Albuquerque UP, Lucena RFP. Métodos e técnicas na pesquisa etnobotânica. Recife: NUPEEA, 2004.

15. Barboza-Silva NC, Regis ACD, Esquibele MA, Espírito JSS, Almeida MZ. Uso de plantas medicinais na comunidade quilombola da Barra II-Bahia, Brasil. Bol. latinoam. Caribe plantas med. aromát. 2012; 11(5): 435-453.

16. Brasil, 2010. Farmacopeia Brasileira, 5 . Agência Nacional de Vigilância Sanitária. Brasília: Ministério da Saúde. RDC 49 de 23 de novembro de 2010.

17. Anvisa, 2008. Instrução normativa $n^{\circ} 5$ de 11 de dezembro de 2008. Lista de Medicamentos Fitoterápicos de Registro Simplificado. Ministério da Saúde. [citado em 2017 nov. 15] Disponível em: http//:e-legis.anvisa.gov.br/leisref/public/showact.php,

18. Carvalho ACB, Lana TN, Perfeito JPS, Silveira D. The Brazilian Market of Herbal Medicinal Products and the Impacts of the New Legislation on Traditional Medicines. J Ethnopharmacol, 2018; 2 (15): 29-35.

19. Anvisa, 2014. Resolução da Diretória Colegia $n^{\circ} 26$ de 13 de maio de 2014. Registro de medicamentos fitoterápicos e o registro e a notificação de produtos tradicionais fitoterápicos. Ministério da Saúde. [citado em 2017 nov. 15] Disponível em: http://bvsms.saude.gov.br/bvs/saudelegis/anvisa/2014/rdc0026_13_05_2014.pdf

20. Jabot - Banco de Dados da Flora Brasileira. JBRJ - Instituto de Pesquisas Jardim Botânico do Rio de Janeiro. [citado em 2017 nov. 15] Disponível em: http://jabot.jbrj.gov.br/

21. APG IV group. An update of the Angiosperm Phylogeny Group classification for the orders and families of flowering plants: APG IV. Bot. J. Linn. Soc. 2016. 181(1), 1-20.

22. Rio de Janeiro. Secretaria Municipal de Saúde e Defesa Civil. Memento Terapêutico do Rio de Janeiro. Gerencia do Programa de Práticas Integrativas e Complementares. - Rio de Janeiro: SMSDC, 2010.

23. Brasil, 2010. Relação Nacional de Plantas Medicinais de Interesse ao SUS. Ministério da Saúde. [citado em 2017 nov. 15] Disponível: http://portal.saude.gov.br/portal/arquivos/pdf/RENISUS_2010.pdf

24. Dolle JM. Para compreender Jean Piaget. Rio de Janeiro: Ed. Zahar, 1975.

25. Keller FS. Teoria do reforço. 6 ed. São Paulo: Editora Pedagógica e Universitária, 1970.

26. Jung CGA. A Natureza da Psique. 2 ed. Petrópolis: Vozes, 1984.

27. Jung CGA. Estudos sobre o simbolismo do si - mesmo. 2 ed. Petrópolis: Vozes, 1986.

28. Freire P. Pedagogia da autonomia: saberes necessários à prática educativa. São Paulo: Paz e Terra, 1996.

29. Secchi L. Políticas públicas: conceitos, esquemas de análise, casos práticos. São Paulo: Cengage Learning, 2010.

30. Queiroz DPN, Lamano-Ferreira APN. Diversidade e Uso de Plantas Cultivadas em Quintais Residenciais Urbanos Localizados na Região da Vila Maria, Zona Norte de São Paulo, SP, Brasil. UNOPAR Cientifica. Rev. Ciênc. Biol. Saúde 2014; 16(4): 299-305.

31. Viganó J, Viganó JA, Cruz-Siva CTA. Utilização de plantas medicinais pela população da região urbana de Três Barras do Paraná. Acta sci., Health sci. 2007; 29 (1): 51-58.

32. Bennett BC, Prance G. Introduced plants in the indigenous pharmacopoeia of Northern South America. Econ. Bot. 2000; 54: 90-102.

33. Silveira PD, Bandeira MAM, Arrais PS. Farmacovigilância e reações adversas às plantas medicinais e fitoterápicos: uma realidade. Rev. Bras. Farmacogn. 2008; 18(4): 618-626.

34. Lourenzani AEBS, Lourenzani WL, Batalha MO. Barreiras e oportunidades na comercialização de plantas medicinais provenientes da agricultura familiar. Inf. Econ. 2004; 34(3): 15-25.

35. Ladio AH, Albuquerque UP. The concept of hybridization and its contribution to urban ethnobiology. Ethnobiol. Conserv. 2014; 3(6): 1-9.

36. Ethur LZ, Jobim JC, Ritter JG, Oliveira G, Trindade B S. Comércio formal e perfil de consumidores 
de plantas medicinais e fitoterápicos no município de Itaqui-RS. Rev. Bras. Plantas Med. 2011;13(2): 121-128.

37. Albuquerque UP. Etnobiologia. Bases Ecológicas e Evolutivas. 1 ed. Recife: NUPPEA, 2013.

38. Ceolin T, Ceolin S, Heck RM, Noguez PT, Zdanski SAD. Relato de experiência do curso de plantas medicinais para profissionais de saúde. Rev. Baiana de Saúde Pública, 2014; 37(2): 501-511

39. Damian AG, Tesser DC, Moretti-Pires RO. Fitoterapia na atenção primária à saúde. Rev. Saúde Pública 2014; 48(3): 541-553.

40. Pey MO. Oficina como modalidade educativa. Perspectiva 1997; 15 (27): 35-63.

41. Pereira AVG, Albiero ALM. A valorização da utilização de plantas medicinais na atenção básica: oficinas de aprendizagem. Arq. Mudi 2015; 19(2): 23-42.

42. Redin E, Streck DR, Zitkoski JJ. Dicionário Paulo Freire. 2 ed. Belo Horizonte: Autêntica, 2010. 